\title{
Reliability analysis for a data flow in event-driven wireless sensor networks using a multiple sending transmission approach
}

\author{
Jun-Bo Wang ${ }^{1 *}$, Jin-Yuan Wang ${ }^{1}$, Ming Chen ${ }^{1}$, Xibin Zhao ${ }^{2}$, Shu-Bin $\mathrm{Si}^{3}$, Lirong Cui ${ }^{4}$, Ling-Ling Cao ${ }^{5}$ \\ and Renhui $\mathrm{Xu}^{6}$
}

\begin{abstract}
Reliability analysis is an important issue in wireless sensor networks (WSNs). This paper aims to study the reliability of a data flow in event-driven wireless sensor networks with multiple sending transmission approach without acknowledgments. Initially, an event-driven wireless sensor network model is described in terms of limited node battery energy and shadowed fading channels. Then, in order to analyze the network reliability, the wireless link reliability and the node energy availability are investigated, respectively. Furthermore, the analytical expressions of the instantaneous network reliability and the mean time to failure (MTTF) are derived. Finally, the simulation results validate the correctness and accuracy of the analytical results.
\end{abstract}

Keywords: Wireless sensor networks; Network reliability; Multiple sending; MTTF

\section{Introduction}

Recent advances in micro-electro-mechanical systems, digital electronics, and wireless communications have led to the emergence of wireless sensor networks (WSNs). A WSN consists of a number of wireless sensor nodes [1]. Each sensor node is a device, equipped with multiple on-board sensing elements, wireless transmitter/receiver modules, computational and power supply elements. Usually, it is characterized by its small size and limited computational and communication capabilities with limited energy supplied by a battery [2]. Indeed, they are deployed in an area of interest to collect data from the environment, process sensed data, and take action accordingly. Typical applications of the WSNs include battlefield surveillance, environmental monitoring, biological detection, smart spaces, and industrial diagnostics [3,4]. Due to the superiority in monitoring spatial phenomena, the WSNs have drawn a lot of attention both in academia and industry.

\footnotetext{
${ }^{*}$ Correspondence: jbwang@seu.edu.cn

${ }^{1}$ National Mobile Communications Research Laboratory, Southeast University, Nanjing 211111, China

Full list of author information is available at the end of the article
}

In the past few years, intensive researches for WSNs have been conducted in many aspects, such as localization [5], synchronization [6,7], deployment [8], and communication protocol [9]. However, it should be emphasized that comparing with other wireless networks, WSNs are more prone to failure due to energy depletion, hardware failure, communication link errors, and so on. Obviously, how to measure the impacts from such network failure is one important issue for practical design and operations, which motivates the research on reliability analysis for WSNs.

So far, reliability analysis has been intensively studied in many traditional wireless communication networks. Chen and Lyu [10] analyzed the end-to-end expected instantaneous reliability for wireless common object request broker architecture (CORBA) networks. Cook and Ramirez-Marquez [11] analyzed the two-terminal reliability for mobile ad hoc networks. They $[12,13]$ also employed Monte Carlo (MC) simulation method to evaluate the reliability of mobile and cluster ad hoc networks, respectively. Dominiak et al. [14] analyzed the terminal-pair (two-terminal) reliability for IEEE 802.16 mesh networks. Liu et al. [15] proposed a more general region failure model to assess the reliability of wireless mesh networks affected from a region failure. Egeland and Engelstad [16] analyzed the $k$-terminal reliability for both 
planned and random wireless mesh networks. However, due to the non-repairable nodes and the limited node battery energy in WSNs, the traditional reliability evaluation methods are not applicable for WSNs. It has actually been reported that the energy constraint is the main factor preventing the full exploitation of WSN technology [17]. So far, only a few researches can be found for the reliability of WSNs in the open literature. AboElFotoh et al. [18] proposed a reliability measure under the assumption that the transmitter/receiver ranges of all sensor nodes are all the same. Obviously, such assumption violates that fact that the real transmitter/receiver ranges usually change with practical wireless link conditions and traffic loads. Shazly et al. [19] further proposed a three-state node reliability model for WSNs reliability analysis. Cheng et al. [20] developed the predictability of collective timeliness for hard network lifetime environments through the worstcase energy consumption analysis. However, the proposed model cannot reflect the relation between the data transmissions and energy consumptions.

This paper will try to analyze the reliability of a data flow in event-driven WSNs with multiple sending transmission approach without acknowledgments. Considering the effects from wireless links, traffic loads, energy consumptions, and node failures, a more rigorous system model is described for a data flow in event-driven WSNs. Based on the proposed system model, wireless link reliability and node energy availability are analyzed respectively. Then, the instantaneous network reliability and the mean time to failure (MTTF) of the data flow in event-driven WSNs are derived.

The remainder of this paper is organized as follows. The system model is introduced in Section 2. In Section 3, the expressions of the node energy availability and the instantaneous network reliability and MTTF of eventdriven wireless networks are derived. Numerical results are presented in Section 4 before conclusions are drawn in Section 5.

\section{System model}

Consider a data flow with a source node, $N$ relay nodes, and a sink node in an event-driven WSN, which is shown in Figure 1. For detailed description about the eventdriven WSN, one can refer to [21,22]. In this paper, the source node generates data packets by sensing events and

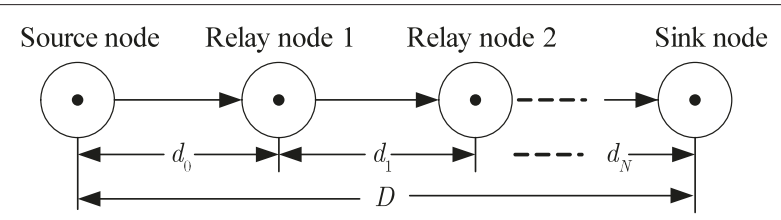

Figure 1 The event-driven wireless sensor network. transits the packets to the sink node through the $N$ relay nodes. To simplify the control mechanism and reduce buffer size at nodes, a multiple sending approach without acknowledgments [23] is adopted as the transmission scheme. Such transmit approach has been proved to be similar to the acknowledgment-based scheme especially under high channel error rate. Except for the sink nodes, all other nodes do not guarantee their functioning over the time and they are normally equipped with low voltage batteries that limit their lifetimes. Without loss of generality, the initial energy available for the source and relay nodes are denoted as $E_{0}^{\text {init }}$ and $E_{n}^{\text {init }}(n=1,2, \ldots, N)$, respectively. When the available energy of a node is less than a threshold level $E_{\text {th }}$, the node will lose its functioning.

To prolong the lifetime, the source node is usually operated in power-saving strategy to save energy. At this strategy, the source node operates either in active mode (i.e., sensing or transmitting) or sleep mode. Since sensing is an energy-consuming operation, the source node generally has its own duty cycle, for instance $1 \%$, which corresponds to $10 \mathrm{~ms}$ sensing event per second. Thus, the energy consumed by the source node to sensing event from time 0 to time $t$ can be given by

$$
E_{0}^{\mathrm{s}}(t)=\alpha P_{0}^{\mathrm{s}} t
$$

where $\alpha$ is the duty cycle, $P_{0}^{\mathrm{s}}$ is the power required by sensing event per second. If some event is detected, the radio module of the source node is turned on and a packet is transmitted to the nearest relay node. Let $K$ denote the number of packet copies sent out by each node in the WSN and assume that totally $M(t)$ events are detected during $[0, t]$, then the energy spent in transmitting packets at the source node can be expressed as

$$
E_{0}^{\mathrm{t}}(t)=\frac{\left(P_{0}^{\mathrm{e}}+P_{0}^{\mathrm{t}}\right) K L M(t)}{r},
$$

where $P_{0}^{\mathrm{e}}$ is the power dissipation of the source node to run the transmitter circuitry, $P_{0}^{\mathrm{t}}$ is the power used by the transmit amplifier (i.e., the transmit power), $L$ is the packet length in bit and $r$ is the transmission rate in bit per second. If no event is detected, the radio module of the source node is kept unavailable. Note that the counting process $M(t), t \geq 0$ which denotes the number of events that are detected by time $t$ is assumed to be a non-homogeneous Poisson process (NHPP) with intensity function $\lambda(t)$. Then $M(t)$ has a Poisson distribution with mean $\Lambda(t)$ which is given as [24]

$$
\operatorname{Pr}\{M(t)=k\}=\frac{(\Lambda(t))^{k}}{k !} \exp (-\Lambda(t)), k=0,1, \ldots,
$$

where $k !$ is the factorial of $k$, and $\Lambda(t)=\int_{0}^{t} \lambda(u) \mathrm{d} u$. 
Specifically, if $\lambda(t)$ does not change over time, i.e., $\lambda(t)=\lambda_{0}$, the counting process $M(t)$ becomes a homogeneous Poisson process and can be written as

$$
\operatorname{Pr}\{M(t)=k\}=\frac{\left(\lambda_{0} t\right)^{k}}{k !} \exp \left(-\lambda_{0} t\right), k=0,1, \ldots
$$

To save energy, the relay nodes also operate in power saving strategy and switch between the sleep mode and the active mode. In most cases, the relay nodes are kept in sleep mode and the radio module is shut off. Under the sleep mode, an analog block stays awake and acts as the radio detector. Once a radio signal is detected, the signal is converted to a control signal which, in turn, is sent to power control electronics to wake up the radio module. With the state-of-art technology, the energy consumed by the analog block is very small. So we only consider the energy consumed while relay nodes are transmitting or receiving in active mode. For the $n$th relay node, assume totally $D_{n}(t)$ events are received during $[0, t]$, the energy spent in receiving packets can be given by

$$
E_{n}^{\mathrm{r}}(t)=\frac{P_{n}^{\mathrm{e}} K L D_{n}(t)}{r} \quad \forall n \in\{1,2, \ldots, N\},
$$

where $P_{n}^{\mathrm{e}}$ is the power dissipation of the $n$th relay node to run the transmitter circuitry. Similar to the source node, for the $n$th relay node, the energy consumed by the packet transmission during $[0, t]$ can be shown as

$$
E_{n}^{\mathrm{t}}(t)=\frac{\left(P_{n}^{\mathrm{e}}+P_{n}^{\mathrm{t}}\right) K L D_{n}(t)}{r} \quad \forall n \in\{1,2, \ldots, N\},
$$

where $P_{n}^{\mathrm{t}}$ is the transmit power of the $n$th relay node.

In the considered data flow, all packets will be transmitted over wireless channels. For facilitating the following description, the source node and the $n$th relay node $(n=$ $1,2, \ldots, N)$ are also called as node 0 and node $n$, respectively. Moreover, the sink node is renamed as node $N+1$. If node $n$ is transmitting a packet to node $n+1$, the receiving signal quality can be measured as the received signal-to-noise ratio (SNR) value, i.e.,

$$
\Gamma_{n}=\frac{P_{n}^{\mathrm{t}} h_{n}}{n_{0}} \quad \forall n \in\{0,1, \ldots, N\}
$$

where $P_{n}^{\mathrm{t}}$ is the transmit power of node $n, n_{0}$ is the background noise power at a receiver, and $h_{n}$ is the wireless channel gain between node $n$ and node $n+1$ and can be calculated in decibels as

$$
\left[h_{n}\right]_{\mathrm{dB}}=[G]_{\mathrm{dB}}-\eta\left[\frac{d_{n}}{d_{\mathrm{ref}}}\right]_{\mathrm{dB}}-[\xi]_{\mathrm{dB}},
$$

where $[x]_{\mathrm{dB}}$ denotes $10 \log _{10} x ; G$ is a dimensionless constant, meaning the measured line-of-sight (LOS) path loss at $d_{\text {ref }}$, which depends on the antenna characteristics and the average channel attenuation; $\eta$ is the path loss exponent for the wireless channel which depends on the terrain and the environment. It may vary between 2 in free space up to 6 in for example urban areas [25]; $d_{\text {ref }}$ is a reference distance for the antenna far-field, $d_{n}$ is the distance between node $n$ and node $n+1 ; \xi$ is the shadow fading and follows a lognormal distribution, i.e., the value of logarithm $[\xi]_{\mathrm{dB}}$ is a normal distribution with mean zero and variance $\sigma_{\xi}^{2}$. To guarantee the receiving accuracy, it is expected that the actual SNR must be no less than the target SNR, i.e.,

$$
\Gamma_{n} \geq \gamma^{\mathrm{t}}
$$

where $\gamma^{\mathrm{t}}$ is the target SNR.

\section{Reliability analysis}

In typical WSN applications, wireless sensor nodes are scattered in large geographical regions and it is not always possible to perform node maintenance after the network deployment. For this reason, all nodes have to adapt their behaviors to the environmental changes. This section will firstly analyze the wireless link reliability and the node energy availability, respectively. Then, the instantaneous network reliability and MTTF are evaluated for the data flow.

\subsection{Wireless link reliability}

For node $n$, one successful transmission can be measured as the received SNR value being no less than the target SNR. Thus, the successful probability of one transmission from node $n$ to node $n+1$ can be defined as

$$
P_{n}^{\text {succ }}=\operatorname{Pr}\left\{\frac{P_{n}^{\mathrm{t}} h_{n}}{n_{0}}>\gamma^{\mathrm{t}}\right\} \quad \forall n \in\{0,1, \ldots, N\}
$$

Substituting (8) into (10), we can obtain

$$
P_{n}^{\text {succ }}=\Phi\left(\sqrt{2} \varphi_{n}\right)=\frac{1}{2}\left(1+\operatorname{erf}\left(\varphi_{n}\right)\right)
$$

where $\Phi(x)=1 / \sqrt{2 \pi} \int_{-\infty}^{x} \exp \left(-t^{2} / 2\right) \mathrm{d} t$ is the cumulative distribution function (CDF) of the standard normal distribution, $\operatorname{erf}(x)=2 / \sqrt{\pi} \int_{0}^{x} \exp \left(-t^{2}\right) \mathrm{d} t$ is the error function, and the parameter $\varphi_{n}$ can be obtain as

$$
\begin{aligned}
\varphi_{n}= & \frac{1}{\sqrt{2} \sigma_{\xi}}\left(\left[P_{n}^{\mathrm{t}}\right]_{\mathrm{dB}}+[G]_{\mathrm{dB}}-\eta\left[\frac{d_{n}}{d_{\mathrm{ref}}}\right]_{\mathrm{dB}}\right. \\
& \left.-\left[n_{0}\right]_{\mathrm{dB}}-\left[\gamma^{\mathrm{t}}\right]_{\mathrm{dB}}\right) .
\end{aligned}
$$

To guarantee the reliable reception, each packet will be transmitted $K$ times. Then the single wireless link 
reliability between node $n$ and node $n+1$ can be obtained as

$$
\begin{aligned}
R_{n}^{\mathrm{L}} & =1-\left(1-P_{n}^{\mathrm{succ}}\right)^{K} \\
& =1-\frac{1}{2^{K}}\left[\operatorname{erfc}\left(\varphi_{n}\right)\right]^{K} \quad \forall n \in\{0,1, \ldots, N\},
\end{aligned}
$$

where $\operatorname{erfc}(x)=1-\operatorname{erf}(x)=2 / \sqrt{\pi} \int_{x}^{\infty} \exp \left(-t^{2}\right) d t$ is the complentary error function.

Furthermore, using (13), the integrated wireless link reliability can be calculated by

$$
R^{\mathrm{L}}=\prod_{n=0}^{N} R_{n}^{\mathrm{L}}=\prod_{n=0}^{N}\left\{1-\frac{1}{2^{K}}\left[\operatorname{erfc}\left(\varphi_{n}\right)\right]^{K}\right\} .
$$

\subsection{Node energy availability}

In the considered WSN, the sensor nodes are powered by low-voltage batteries. If the stored energy is depleted, the node is an energy-unavailable node and thus loses its functioning. Based on the energy stored in a node, the node energy availability for source node and relay nodes will be discussed in the following subsections.

\subsubsection{Source node energy availability}

For the source node (node 0 ), the residual energy at time $t$ can be obtained as

$$
\begin{aligned}
E_{0}^{\mathrm{re}}(t) & =E_{0}^{\mathrm{init}}-\left(E_{0}^{\mathrm{s}}(t)+E_{0}^{\mathrm{t}}(t)\right) \\
& =E_{0}^{\mathrm{init}}-\alpha P_{0}^{\mathrm{s}} t-\frac{\left(P_{0}^{\mathrm{e}}+P_{0}^{\mathrm{t}}\right) K L M(t)}{r} .
\end{aligned}
$$

When $E_{0}^{\text {re }}(t)$ is less than a threshold $E_{\text {th }}$, the sensor node cannot work normally. Assume that symbol $\mathrm{A}_{0}$ is the state that the source node is energy available at time $t$ and then the energy availability of the source node at time $t$ is given as

$$
\begin{aligned}
\operatorname{Pr}\left\{\mathrm{A}_{0}\right\} & =\operatorname{Pr}\left\{E_{0}^{\mathrm{re}}(t) \geq E_{\mathrm{th}}\right\} \\
& =\operatorname{Pr}\left\{M(t) \leq \frac{\left(E_{0}^{\text {init }}-\alpha P_{0}^{\mathrm{s}} t-E_{\mathrm{th}}\right) r}{\left(P_{0}^{\mathrm{e}}+P_{0}^{\mathrm{t}}\right) K L}\right\} .
\end{aligned}
$$

According to (3), the energy availability of the source node at time $t$ can be measured as

$$
\operatorname{Pr}\left\{\mathrm{A}_{0}\right\}=\left\{\begin{array}{cc}
\sum_{k=0}^{M_{0}} \frac{(\Lambda(t))^{k}}{k !} \exp (-\Lambda(t)), & M_{0} \geq 0, \\
0, & M_{0}<0,
\end{array}\right.
$$

where $M_{0}$ is an integer and can be obtained as

$$
M_{0}=\left\lfloor\frac{E_{0}^{\mathrm{init}}-\alpha P_{0}^{\mathrm{s}} t-E_{\mathrm{th}}}{\left(P_{0}^{\mathrm{e}}+P_{0}^{\mathrm{t}}\right) K L}\right\rfloor .
$$

In (18), $\lfloor x\rfloor=\min \{m \in Z \mid m \leq x\}$ is the largest integer less than $x$. Using the upper incomplete gamma function defined in [26], (17) can be further modified into

$$
\operatorname{Pr}\left\{\mathrm{A}_{0}\right\}=\left\{\begin{array}{cc}
\frac{1}{M_{0} !} \Gamma\left(M_{0}+1, \Lambda(t)\right), & M_{0} \geq 0, \\
0, & M_{0}<0,
\end{array}\right.
$$

where the function $\Gamma(\mu, x)=\int_{x}^{\infty} e^{-\tau} \tau^{\mu-1} \mathrm{~d} \tau$ is the upper incomplete gamma function.

Specifically, if $\lambda(t)$ is a constant $\lambda_{0}$, (19) can be transformed into

$$
\operatorname{Pr}\left\{\mathrm{A}_{0}\right\}=\left\{\begin{array}{cc}
\frac{1}{M_{0} !} \Gamma\left(M_{0}+1, \lambda_{0} t\right), & M_{0} \geq 0, \\
0, & M_{0}<0 .
\end{array}\right.
$$

To reduce the computation complexity, we further analyze the probability in (19) and obtain Proposition 1.

Proposition 1. At time $t$, if the value of $\Lambda(t)$ is sufficiently large (e.g., the value of $\Lambda(t)$ is greater than 10), then the energy availability of source node can be expressed approximately as follows:

$\operatorname{Pr}\left\{\mathrm{A}_{0}\right\} \approx\left\{\begin{array}{cc}\frac{1}{2}\left[1+\operatorname{erf}\left(\frac{M_{0}+0.5-\Lambda(t)}{\sqrt{2 \Lambda(t)}}\right)\right], & M_{0} \geq 0, \\ 0, & M_{0}<0 .\end{array}\right.$

Proof. See Appendix 1.

\subsubsection{Relay node energy availability}

Similar to the source node, the residual energy for the $n$ th, $\forall n \in\{1,2, \ldots, N\}$ relay node (node $n$ ) at time $t$ can be obtained as

$$
\begin{aligned}
E_{n}^{\mathrm{re}}(t) & =E_{n}^{\mathrm{init}}-\left(E_{n}^{\mathrm{r}}(t)+E_{n}^{\mathrm{t}}(t)\right) \\
& =E_{n}^{\mathrm{init}}-\frac{\left(2 P_{n}^{\mathrm{e}}+P_{n}^{\mathrm{t}}\right) K L D_{n}(t)}{r},
\end{aligned}
$$

where $D_{n}(t)$ is a random variable which describes the number of packets that are successfully received by node $n$ during $[0, t]$. Define $\mathrm{A}_{n}, \forall n \in\{1,2, \ldots, N\}$ as a state that node $n$ is energy available at time $t$, and then the energy availability of relay node is defined as

$$
\begin{aligned}
\operatorname{Pr}\left\{\mathrm{A}_{n}\right\} & =\operatorname{Pr}\left\{E_{n}^{\mathrm{re}}(t) \geq E_{\mathrm{th}}\right\} \\
& =\operatorname{Pr}\left\{D_{n}(t) \leq \frac{r\left(E_{n}^{\mathrm{init}}-E_{\mathrm{th}}\right)}{\left(2 P_{n}^{\mathrm{e}}+P_{n}^{\mathrm{t}}\right) K L}\right\} .
\end{aligned}
$$

Obviously, to obtain the energy availability of the $n$th relay node, $D_{n}(t)$ should be measured firstly. According to 
(13), affected by the channel fading, the packets may not be received successfully. Therefore, $D_{n}(t)$ should be a discrete random variable. The probability distribution of random variable $D_{n}(t)$ can be described as (see Appendix 2 for the detailed derivation)

$$
\begin{aligned}
\operatorname{Pr}\left\{D_{n}(t)=\right. & \left.i \mid \mathrm{A}_{0} \mathrm{~A}_{1} \ldots \mathrm{A}_{n-1}\right\}=\sum_{k=i}^{\infty}\left(\begin{array}{l}
k \\
i
\end{array}\right)\left(R_{0-n}^{\mathrm{L}}\right)^{i} \\
& \times\left(1-R_{0-n}^{\mathrm{L}}\right)^{k-i} \frac{(\Lambda(t))^{k}}{k !} \exp (-\Lambda(t)), \\
i= & 0,1, \ldots
\end{aligned}
$$

where $\left(\begin{array}{l}k \\ i\end{array}\right)=k ! /[i !(k-i) !]$ for $k \geq 0, i \geq 0, k \geq i$ denotes the binomial coefficients [27], and $R_{0-n}^{\mathrm{L}} \triangleq \prod_{j=0}^{n-1} R_{j}^{\mathrm{L}}$.

Substituting (24) into (23), the probability of the $n$th relay node which is energy available can be given by

$$
\begin{aligned}
\operatorname{Pr}\left\{\mathrm{A}_{n} \mid \mathrm{A}_{0} \mathrm{~A}_{1} \ldots \mathrm{A}_{n-1}\right\}= & \operatorname{Pr}\left\{D_{n}(t) \leq M_{n} \mid \mathrm{A}_{0} \mathrm{~A}_{1} \ldots \mathrm{A}_{n-1}\right\} \\
= & \sum_{i=0}^{M_{n}} \operatorname{Pr}\left\{D_{n}(t)=i \mid \mathrm{A}_{0} \mathrm{~A}_{1} \ldots \mathrm{A}_{n-1}\right\} \\
= & \sum_{i=0}^{M_{n}} \sum_{k=i}^{\infty}\left(\begin{array}{l}
k \\
i
\end{array}\right)\left(R_{0-n}^{\mathrm{L}}\right)^{i}\left(1-R_{0-n}^{\mathrm{L}}\right)^{k-i} \\
& \times \frac{(\Lambda(t))^{k}}{k !} \exp (-\Lambda(t)),
\end{aligned}
$$

where $M_{n}$ can be obtained by

$$
M_{n}=\left\lfloor\frac{\left(E_{n}^{\mathrm{init}}-E_{\mathrm{th}}\right) r}{\left(2 P_{n}^{\mathrm{e}}+P_{n}^{\mathrm{t}}\right) K L}\right\rfloor, \forall n \in\{1,2, \ldots, N\} .
$$

Specifically, if $\lambda(t)$ is a constant $\lambda_{0}$, the probability of the $n$th relay node which is energy available can be given by

$$
\begin{aligned}
\operatorname{Pr}\left\{\mathrm{A}_{n} \mid \mathrm{A}_{0}, \mathrm{~A}_{1}, \ldots, \mathrm{A}_{n-1}\right\}= & \sum_{i=0}^{M_{n}} \sum_{k=i}^{\infty}\left(\begin{array}{l}
k \\
i
\end{array}\right)\left(R_{0-n}^{\mathrm{L}}\right)^{i} \\
& \times\left(1-R_{0-n}^{\mathrm{L}}\right)^{k-i} \frac{\left(\lambda_{0} t\right)^{k}}{k !} \\
& \times \exp \left(-\lambda_{0} t\right) .
\end{aligned}
$$

Furthermore, exchanging the order of summation in (25) and then using the relationship between the regularized incomplete beta function and the cumulative distribution function of a binomial variable $[28,29]$, the probability of the $n$th relay node is energy available can be calculated by

$$
\begin{aligned}
\operatorname{Pr}\left\{\mathrm{A}_{n} \mid \mathrm{A}_{0} \mathrm{~A}_{1} \ldots \mathrm{A}_{n-1}\right\}= & \Gamma\left(M_{n}+1, \Lambda(t)\right) \\
& +\sum_{k=M_{n}+1}^{\infty} \frac{(\Lambda(t))^{k}}{k !} \exp (-\Lambda(t)) \\
& \times \mathrm{I}_{1-R_{0-n}^{\mathrm{L}}}\left(k-M_{n}, M_{n}+1\right),
\end{aligned}
$$

where $\mathrm{I}_{x}(a, b)=\left[\int_{0}^{x} t^{a-1}(1-t)^{b-1} \mathrm{~d} t\right] /\left[\int_{0}^{1} t^{a-1}(1-t)^{b-1} \mathrm{~d} t\right]$ is the regularized incomplete beta function.

Specifically, if $\lambda(t)$ is a constant $\lambda_{0}$, the probability of the $n$th relay node is energy available can be given by

$$
\begin{aligned}
\operatorname{Pr}\left\{\mathrm{A}_{n} \mid \mathrm{A}_{0} \mathrm{~A}_{1} \ldots \mathrm{A}_{n-1}\right\}= & \Gamma\left(M_{n}+1, \lambda_{0} t\right) \\
& +\sum_{k=M_{n}+1}^{\infty} \frac{\left(\lambda_{0} t\right)^{k}}{k !} \exp \left(-\lambda_{0} t\right) \mathrm{I}_{1-R_{0-n}^{\mathrm{L}}} \\
& \times\left(k-M_{n}, M_{n}+1\right) .
\end{aligned}
$$

To reduce the computation complexity, we further analyze the probability in (25) and obtain Proposition 2.

Proposition 2. The energy availability of the $n t h, \forall n \in$ $\{1,2, \ldots, N\}$ relay node can be expressed approximately as follows:

$$
\operatorname{Pr}\left\{\mathrm{A}_{n} \mid \mathrm{A}_{0} \mathrm{~A}_{1} \ldots \mathrm{A}_{n-1}\right\} \approx \Gamma\left(M_{n}^{\prime}+1, \Lambda(t)\right),
$$

where

$$
M_{n}^{\prime}=\left\lfloor\frac{r\left(E_{n}^{\mathrm{init}}-E_{\mathrm{th}}\right)}{\left(2 P_{n}^{\mathrm{e}}+P_{n}^{\mathrm{t}}\right) K L R_{0-n}^{\mathrm{L}}}\right\rfloor .
$$

Note that if the value of $\Lambda(t)$ at time $t$ is sufficiently large (e.g., the value of $\Lambda(t)$ is greater than 10), the energy availability of the $n t h, \forall n \in\{1,2, \ldots, N\}$ relay node can further be expressed approximately as follows:

$$
\operatorname{Pr}\left\{\mathrm{A}_{n} \mid \mathrm{A}_{0} \mathrm{~A}_{1} \ldots \mathrm{A}_{n-1}\right\} \approx \frac{1}{2}\left[1+\operatorname{erf}\left(\frac{M_{n}^{\prime}+0.5-\Lambda(t)}{\sqrt{2 \Lambda(t)}}\right)\right] .
$$

\section{Proof. See Appendix 3.}

\subsection{Instantaneous network reliability}

Taking account of the above factors of wireless link reliability and node energy availability, the instantaneous network reliability in this paper can be defined as

$$
\begin{aligned}
R_{\text {sys }}(t) & =\operatorname{Pr}\left\{\mathrm{A}_{0} \mathrm{~A}_{1} \ldots \mathrm{A}_{N-1} \mathrm{~A}_{N}\right\} \\
& =\operatorname{Pr}\left\{\mathrm{A}_{0}\right\} \prod_{n=1}^{N} \operatorname{Pr}\left\{\mathrm{A}_{n} \mid \mathrm{A}_{0} \mathrm{~A}_{1} \ldots \mathrm{A}_{n-1}\right\} .
\end{aligned}
$$


Accordingly, based on the total probability formula, using (19) and (25), the instantaneous network reliability can be calculated by

$$
\begin{aligned}
R_{\text {sys }}(t)= & \frac{1}{M_{0} !} \Gamma\left(M_{0}+1, \Lambda(t)\right) \\
& \times \prod_{n=1}^{N}\left\{\sum_{i=0}^{M_{n}} \sum_{k=i}^{\infty}\left(\begin{array}{l}
k \\
i
\end{array}\right)\left(R_{0-n}^{\mathrm{L}}\right)^{i}\left(1-R_{0-n}^{\mathrm{L}}\right)^{k-i}\right. \\
& \left.\times \frac{(\Lambda(t))^{k}}{k !} \exp (-\Lambda(t))\right\}
\end{aligned}
$$

Specifically, according to (20) and (27), if $\lambda(t)$ is a constant $\lambda_{0}$, the instantaneous network reliability can be calculated by

$$
\begin{aligned}
R_{\text {sys }}(t)= & \frac{1}{M_{0} !} \Gamma\left(M_{0}+1, \lambda_{0} t\right) \\
& \times \prod_{n=1}^{N}\left\{\sum_{i=0}^{M_{n}} \sum_{k=i}^{\infty}\left(\begin{array}{l}
k \\
i
\end{array}\right)\left(R_{0-n}^{\mathrm{L}}\right)^{i}\left(1-R_{0-n}^{\mathrm{L}}\right)^{k-i}\right. \\
& \left.\times \frac{\left(\lambda_{0} t\right)^{k}}{k !} \exp \left(-\lambda_{0} t\right)\right\} .
\end{aligned}
$$

According to Proposition 2, the instantaneous network reliability (34) can be further approximately expressed as

$$
R_{\text {sys }}(t) \approx \prod_{n=0}^{N} \frac{1}{M_{n}^{\prime} !} \Gamma\left(M_{n}^{\prime}+1, \Lambda(t)\right),
$$

where $M_{0}^{\prime}=M_{0}$.

\subsection{MTTF}

The MTTF measures the average time to failures with the modeling assumption that the failed system is not repaired. In this system, the values of MTTF can be obtained by integrating the instantaneous network reliability function from zero to infinity:

$$
\operatorname{MTTF}=\int_{0}^{\infty} R_{\text {sys }}(t) \mathrm{d} t
$$

Moreover, using (36), MTTF can be calculated approximately as

$$
\operatorname{MTTF} \approx \int_{0}^{\infty} \prod_{n=0}^{N}\left\{\frac{1}{M_{n}^{\prime} !} \Gamma\left(M_{n}^{\prime}+1, \Lambda(t)\right)\right\} \mathrm{d} t .
$$

\section{Numerical results}

In this section, both the Monte Carlo (MC) simulation results and theoretical results will be presented. Here, the accuracy of the derived expressions of wireless link reliability, the instantaneous network reliability, and MTTF will be verified, and the impacts of the number of relay nodes will be discussed.

Here, an event-driven WSN consisting of $N$ uniformly placed relay nodes in a $D$-meter-long linear region is used as a test system. To simplify the simulation, assume that the source node and all relay nodes have the same initial energy (i.e., $E_{0}^{\text {init }}=E_{1}^{\text {init }}=\cdots=E_{N}^{\text {init }} \triangleq E^{\text {init }}$ ) and run the transmitter circuitry with the same power (i.e., $P_{0}^{\mathrm{e}}=$ $\left.P_{1}^{\mathrm{e}}=\cdots=P_{N}^{\mathrm{e}} \triangleq P^{\mathrm{e}}\right)$. Moreover, the transmit power of the source node and all relay nodes are also supposed to be the same (i.e., $P_{0}^{\mathrm{t}}=P_{1}^{\mathrm{t}}=\cdots=P_{N}^{\mathrm{t}} \triangleq P^{\mathrm{t}}$ ). In addition, the main simulation parameters are listed in Table 1.

Figure 2 illustrates the wireless link reliability versus transmit power with different numbers of the relay nodes. As the figure clearly illustrates, with the increase of the transmit power, the larger received SNR at each receiver occurs, which results in the increase of wireless link reliability. Simultaneously, the results in Figure 2 indicate that the relay node number affects the wireless link reliability significantly. Specifically, the wireless link reliability with $N=5$ is much higher than that with $N=3$. The reason is that a larger relay node number will result in smaller distance between two adjacent nodes which will further result in higher link reliability. In addition, it can be observed from Figure 2 that the simulation results of wireless link reliability match with the theoretical results very well.

Figure 3 illustrates the instantaneous network reliability versus time with different numbers of the relay nodes for $R^{\mathrm{L}}=0.7$. In this figure, not only the theoretical results and simulation results are shown, but also the approximate

\section{Table 1 Main simulation parameters}

\begin{tabular}{lll}
\hline Parameters & Symbol & Value \\
\hline Packet copies & $K$ & 1 \\
Intensity function for NHPP models & $\lambda(t)$ & 1 \\
The initial energy of all nodes & $E^{\text {init }}$ & $0.01 \mathrm{~J}$ \\
Threshold level & $E_{\text {th }}$ & $0 \mathrm{~J}$ \\
Duty cycle & $\alpha$ & 0.01 \\
Power required by sensing event per second & $P_{0}^{\mathrm{s}}$ & $0.1 \mathrm{~mW}$ \\
Power dissipation to run transmitter circuitry & $P^{\mathrm{e}}$ & $0.1 \mathrm{~mW}$ \\
Length of the linear region & $D$ & $180 \mathrm{~m}$ \\
Transmission rate & $r$ & $2.5 \times 10^{5} \mathrm{bit} / \mathrm{s}$ \\
Packet length & $L$ & $5,000 \mathrm{bits}$ \\
Power of background noise & $n_{0}$ & $4 \times 10^{-14} \mathrm{~W}$ \\
Target SNR & $\gamma^{\mathrm{t}}$ & $6 \mathrm{~dB}$ \\
Variance of shadow fading & $\sigma_{\xi}^{2}$ & $8 \mathrm{~dB}$ \\
Path loss exponent & $\eta$ & 3.71 \\
LOS path loss at $d_{\text {ref }}$ & $G$ & $-31.54 \mathrm{~dB}$ \\
\hline
\end{tabular}




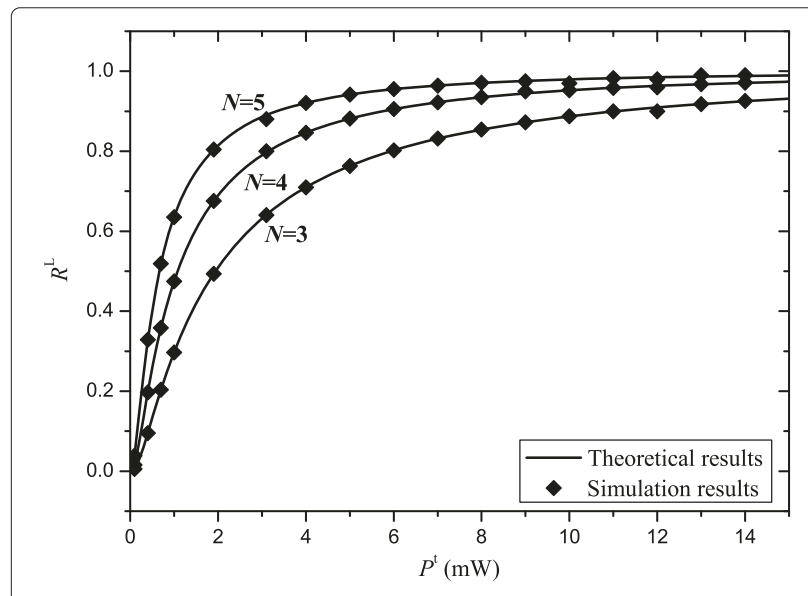

Figure 2 Wireless link reliability versus transmit power with different $N$.

results are depicted. As the figure clearly illustrates, the instantaneous network reliability at the beginning remains the same. It is because the amount of energy of sensor nodes in the initial stage is enough to guarantee the normal work of the sensor nodes. After working for some time, the residual energy of nodes is getting smaller and smaller, which results in the lower probability of sensor nodes to work properly. Then, the network reliability falls sharply. Accordingly, the instantaneous network reliability decreases with the time increasing. Simultaneously, more relay nodes may lead to the higher network reliability. It is because when you want to get the same wireless link reliability (e.g., $R^{\mathrm{L}}$ is 0.7 ), larger relay node number may result in lower transmit power of each node required as shown in Figure 2. It means all the nodes may consume less energy. For this reason, the energy availability of each relay node is increased with the number of the relay nodes. Therefore, the network reliability increases as well.

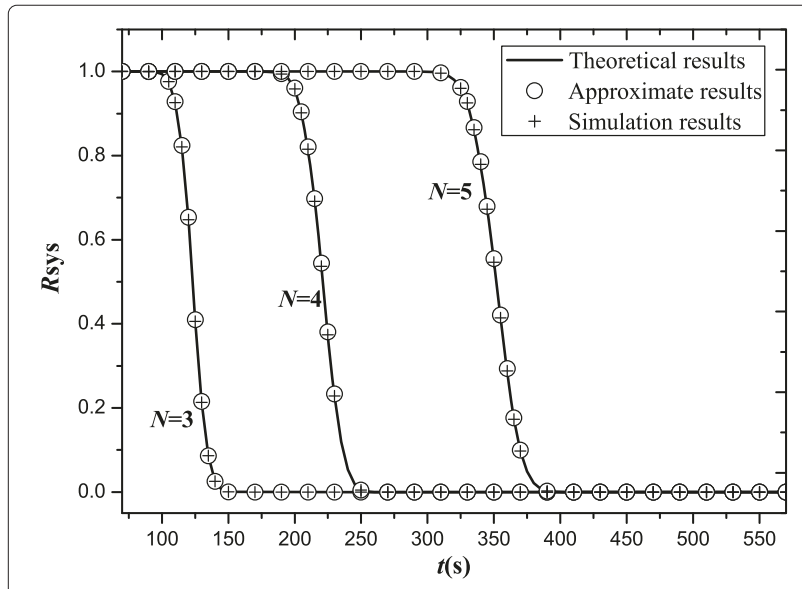

Figure 3 Instantaneous network reliability versus time with different $N$.
Figure 4 gives another different perspective of the same results about instantaneous network reliability, as it shows the instantaneous network reliability versus time with different wireless link reliabilities for $N=4$. Apparently, the instantaneous network reliability decreases along with the increase of the time which has been explained in the above paragraphs. It can also be observed that a high reliable link may result in the a low reliable network. It is because higher link reliability may require the higher transmit power which will result in higher node energy consumption, thus leading to the decrease of node energy availability. Consequently, if you want to get higher link reliability in a WSN, it may result in lower network reliability. Furthermore, from Figures 3 and 4, it can be easily observed that not only the simulation results of network reliability match very well with the theoretical results but also the approximate results match well with the theoretical results.

Figure 5 demonstrates the network MTTF of the wireless sensor network versus wireless link reliability with different relay node number. Obviously, with the increase of the wireless link reliability, the sensor node will require the larger transmit power as shown in Figure 2, thus leading to the decreased value of MTTF. Simultaneously, the more relay nodes may result in the higher network reliability as shown in Figure 3, which results in the greater values of MTTF. Moreover, the values of MTTF tend to be 0 when the link reliability tends to be 1 as shown in Figure 5. It is because in order to guarantee that the link reliability is equal to 1 , the required transmit power tends to be infinite, which results in the lifetime of each node which tends to be 0 . Furthermore, from Figure 5, it can be easily observed that not only the simulation results of MTTF match very well with the theoretical results but also the approximate results match well with the theoretical results.

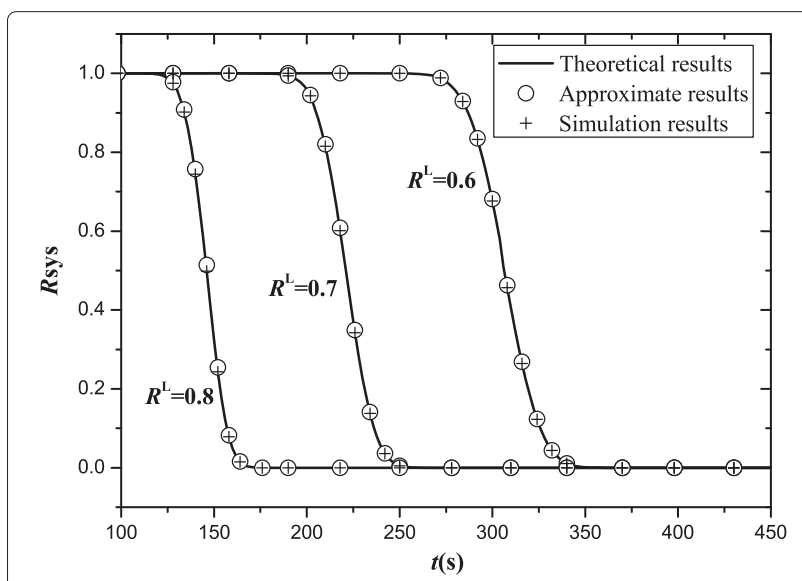

Figure 4 Instantaneous network reliability versus time with different $R^{\mathrm{L}}$. 


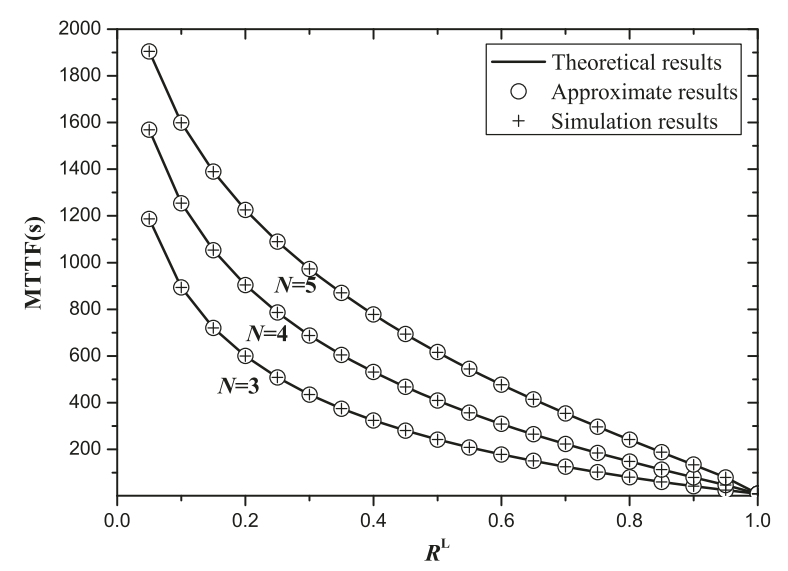

Figure 5 MTTF versus wireless link reliability with different $N$.

\section{Conclusions}

Sensing the environment and sending the sensed information to a sink node are two primary jobs of a WSN. These jobs are required to be done with greater reliability because some major decisions depend on the information collected from WSNs. However, sensors are typically powered through batteries in WSNs and it has been reported that the energy constraint is the main factor preventing the full exploitation of WSN technology. So, reliability evaluation is a critical step for the design of the WSNs. Based on energy-based reliability model, this paper investigates the system performance in an eventdriven wireless sensor network with a multiple sending approach without acknowledgments. A system model is established including sensor energy consumption model and the wireless link model. Then the wireless link reliability, node energy availability, instantaneous network, and MTTF are investigated in this paper. However, the node energy availability expression and the system instantaneous reliability expression are not in closed form, thereby making calculation cumbersome. To bypass this problem, two propositions are developed which make it possible to calculate the node energy availability and the system instantaneous reliability easier. The simulation results show that the analytical expressions are accurate enough. Furthermore, the results are useful in designing a WSN to obtain good network performance. For future work, the reliability analysis of a data flow in event-driven WSN with acknowledgment-based transmission scheme and the reliability evaluation of the WSNs with a random node distribution will be investigated.

\section{Appendices}

Appendix 1

\section{Proof of proposition 1}

Proof. It can be obviously found from (17) that the energy availability of source node at time $t$ can be regarded as the CDF of a Poisson distribution with mean $\Lambda(t)$ and variance $\Lambda(t)$ at $M_{0}$. According to [30], for sufficiently large values of $\Lambda(t)$ (say $\Lambda(t)>1,000$ ), the normal distribution with mean $\Lambda(t)$ and variance $\Lambda(t)$ is an excellent approximation to the Poisson distribution. If $\Lambda(t)$ is greater than about 10, then the normal distribution is a good approximation if an appropriate continuity correction is performed, i.e., $\operatorname{Pr}\{X \leq x\}$ is replaced by $\operatorname{Pr}\{X \leq x+0.5\}$. Therefore, the energy availability of source node can be approximately expressed as

$$
\operatorname{Pr}\left\{\mathrm{A}_{0}\right\} \approx\left\{\begin{array}{cc}
\Phi\left\{\frac{M_{0}+0.5-\Lambda(t)}{\sqrt{\Lambda(t)}}\right\}, & M_{0} \geq 0, \\
0, & M_{0}<0 .
\end{array}\right.
$$

Using the relationship between $\Phi(x)$ and $\operatorname{erf}(x)$, (39) can be further described as

$\operatorname{Pr}\left\{\mathrm{A}_{0}\right\} \approx\left\{\begin{array}{cc}\frac{1}{2}\left[1+\operatorname{erf}\left(\frac{M_{0}+0.5-\Lambda(t)}{\sqrt{2 \Lambda(t)}}\right)\right], & M_{0} \geq 0 \\ 0, & M_{0}<0 .\end{array}\right.$

Proposition 1 is proved.

\section{Appendix 2}

\section{Derivation of the probability distribution of random variable} $D_{n}(t)$

The probability distribution of random variable $D_{n}(t)$ will be deduced in this appendix.

Firstly, $D_{1}(t)$ which describes the number of packets that are received by the first relay node during $[0, t]$ will be considered. Obviously, the packets that are received successfully by the first relay node during $[0, t]$ are decided by both the state of the source node at time $t$ and the reliability of the link between the source node and the first relay node. In this paper, we just concern the probability distribution of $D_{1}(t)$ when the source node is available. Hence, the notation $D_{1}(t) \mid \mathrm{A}_{0}$ is employed to indicate the $D_{1}(t)$ when the source node is available. According to (13), the probability of the first relay node successfully receiving one packet from the source node is $R_{0}^{\mathrm{L}}$. Therefore, conditionally on $M(t)=k$, the process that the first relay node receives packets is a $k$ Bernoulli trial during $[0, t]$ and the probability of success in this Bernoulli trial is $R_{0}^{\mathrm{L}}$. As a result, the random variable $D_{1}^{\prime}=D_{1}(t) \mid \mathrm{A}_{0}$ follows the binomial distribution with parameters $M(t)=k$ and $R_{0}^{\mathrm{L}}$, namely,

$$
D_{1}^{\prime} \sim B\left(M(t)=k, R_{0}^{\mathrm{L}}\right) .
$$


The probability distribution of such a random variable can be given by

$$
\begin{gathered}
\operatorname{Pr}\left\{D_{1}(t)=i \mid M(t)=k, \mathrm{~A}_{0}\right\} \\
=\left\{\begin{array}{cc}
\left(\begin{array}{l}
k \\
i
\end{array}\right)\left(R_{0}^{\mathrm{L}}\right)^{i}\left(1-R_{0}^{\mathrm{L}}\right)^{k-i}, & i=0,1, \ldots, k, \\
0, & \text { others, }
\end{array}\right.
\end{gathered}
$$

where $\left(\begin{array}{l}k \\ i\end{array}\right)$ can be calculated by

$$
\left(\begin{array}{l}
k \\
i
\end{array}\right)=\frac{k !}{i !(k-i) !} \text {. }
$$

According to the formula of total probability and using (3) and (42), the probability distribution of random variable $D_{1}(t) \mid \mathrm{A}_{0}$ can be described as

$$
\begin{aligned}
\operatorname{Pr}\left\{D_{1}(t)=i \mid \mathrm{A}_{0}\right\}= & \sum_{k=i}^{\infty}\left(\begin{array}{l}
k \\
i
\end{array}\right)\left(R_{0}^{\mathrm{L}}\right)^{i}\left(1-R_{0}^{\mathrm{L}}\right)^{k-i} \\
& \times \frac{(\Lambda(t))^{k}}{k !} \exp (-\Lambda(t)), \\
i= & 0,1, \ldots
\end{aligned}
$$

Furthermore, for the second relay node, conditional on $D_{1}^{\prime}=i$, the random variable $D_{2}^{\prime}=D_{2}(t) \mid \mathrm{A}_{0} \mathrm{~A}_{1}$ is also a binomial variable with parameters $D_{1}^{\prime}=i$ and $R_{1}^{\mathrm{L}}$, namely,

$$
D_{2}^{\prime} \sim B\left(D_{1}^{\prime}=i, R_{1}^{\mathrm{L}}\right)
$$

According to the [28], $D_{2}^{\prime}$ is a simple binomial variable with parameters $M(t)=k$ and $\prod_{i=0}^{1} R_{i}^{\mathrm{L}}$, namely,

$$
D_{2}^{\prime} \sim B\left(M(t)=k, \prod_{i=0}^{1} R_{i}^{\mathrm{L}}\right) .
$$

The probability distribution of such a random variable can be given by

$$
\begin{aligned}
\operatorname{Pr} & \left\{D_{2}(t)=i \mid M(t)=k, \mathrm{~A}_{0} \mathrm{~A}_{1}\right\} \\
=\left\{\left(\begin{array}{l}
k \\
i
\end{array}\right)\left[\prod_{i=0}^{1} R_{i}^{\mathrm{L}}\right]^{i}\left[\begin{array}{c}
1 \\
0,
\end{array} \prod_{i=0}^{1} R_{i}^{\mathrm{L}}\right]^{k-i},\right. & i=0,1, \ldots, k,
\end{aligned}
$$

Hence, using the formula of total probability, the probability distribution of random variable $D_{2}(t) \mid \mathrm{A}_{0} \mathrm{~A}_{1}$ can be described by

$$
\begin{aligned}
\operatorname{Pr}\left\{D_{2}(t)=i \mid \mathrm{A}_{0} \mathrm{~A}_{1}\right\}= & \sum_{k=i}^{\infty}\left(\begin{array}{l}
k \\
i
\end{array}\right)\left[\prod_{i=0}^{1} R_{i}^{\mathrm{L}}\right]^{i}\left[1-\prod_{i=0}^{1} R_{i}^{\mathrm{L}}\right]^{k-i} \\
& \times \frac{(\Lambda(t))^{k}}{k !} \exp (-\Lambda(t))
\end{aligned}
$$$$
i=0,1, \ldots
$$

Without loss of generality, for the $n$ th, $\forall n=3, \ldots, N$ relay node, we have

$$
\begin{aligned}
& \operatorname{Pr}\left\{D_{n}(t)=i \mid M(t)=k, \mathrm{~A}_{0} \mathrm{~A}_{1} \ldots \mathrm{A}_{n-1}\right\} \\
&=\left\{\begin{array}{cc}
\left(\begin{array}{l}
k \\
i
\end{array}\right)\left(R_{0-n}^{\mathrm{L}}\right)^{i}\left(1-R_{0-n}^{\mathrm{L}}\right)^{k-i}, & i=0,1, \ldots, k, \\
0, & \text { others, }
\end{array}\right.
\end{aligned}
$$

where

$$
R_{0-n}^{\mathrm{L}} \triangleq \prod_{j=0}^{n-1} R_{j}^{\mathrm{L}}
$$

Then, the probability distribution of random variable $D_{n}(t) \mid \mathrm{A}_{0} \mathrm{~A}_{1} \ldots \mathrm{A}_{n-1}, \forall n \in\{3, \ldots, N\}$ can be described as

$$
\begin{aligned}
\operatorname{Pr} & \left\{D_{n}(t)=i \mid \mathrm{A}_{0} \mathrm{~A}_{1} \ldots \mathrm{A}_{n-1}\right\} \\
& =\sum_{k=i}^{\infty}\left(\begin{array}{l}
k \\
i
\end{array}\right)\left(R_{0-n}^{\mathrm{L}}\right)^{i}\left(1-R_{0-n}^{\mathrm{L}}\right)^{k-i} \frac{(\Lambda(t))^{k}}{k !} \exp (-\Lambda(t)), \\
i & =0,1, \ldots
\end{aligned}
$$

According to (44), (48), and (51), for the $n$ th, $\forall n \in$ $\{1,2, \ldots, N\}$ relay node, we can obtain

$$
\begin{aligned}
\operatorname{Pr}\left\{D_{n}(t)=i \mid \mathrm{A}_{0} \mathrm{~A}_{1} \ldots \mathrm{A}_{n-1}\right\}= & \sum_{k=i}^{\infty}\left(\begin{array}{l}
k \\
i
\end{array}\right)\left(R_{0-n}^{\mathrm{L}}\right)^{i}\left(1-R_{0-n}^{\mathrm{L}}\right)^{k-i} \\
& \times \frac{(\Lambda(t))^{k}}{k !} \exp (-\Lambda(t)), \\
i= & 0,1, \ldots
\end{aligned}
$$

\section{Appendix 3}

\section{Proof of proposition 2}

Proof. Firstly, the relationship between the random variable $M(t)$ and the random variable $D_{n}(t), \forall n \in$ $\{1,2, \ldots, N\}$ will be discussed. It has been known that $M(t)$ denotes the number of packets that are detected by the source node during $[0, t]$ and it is also equal to the number of packets that are sent out from the source node during $[0, t] . D_{n}(t)$ denotes the number of packets that are successfully received by the $n$th relay node during $[0, t]$. $R_{n}^{\mathrm{L}}, \forall n \in\{0,1, \ldots, N\}$ is the link reliability between node $n$ and node $n+1$. Moreover, it has been assumed that it does not concern propagation delay. Thus, for the first relay node, assume the source node is energy available at time $t$, then it can be obtained that

$$
D_{1}(t) \mid \mathrm{A}_{0} \approx R_{0}^{\mathrm{L}} M(t) .
$$


Without loss of generality, for the $n$ th, $\forall n \in\{2,3, \ldots, N\}$ relay node, we can obtain

$$
D_{n}(t)\left|\mathrm{A}_{0} \mathrm{~A}_{1} \ldots \mathrm{A}_{n-1} \approx R_{n-1}^{\mathrm{L}} D_{n-1}(t)\right| \mathrm{A}_{0} \mathrm{~A}_{1} \ldots \mathrm{A}_{n-2} .
$$

Further, according to (53) and (54), it can be obtained that

$$
D_{n}(t) \mid \mathrm{A}_{0} \mathrm{~A}_{1} \ldots \mathrm{A}_{n-1} \approx R_{0-n}^{\mathrm{L}} M(t), \forall n \in\{1,2 \ldots, N\} .
$$

Hence, substituting (3) and (55) into (23), the probability of the $n$ th, $\forall n \in\{1,2, \ldots, N\}$ relay node which is energy available can be given by

$$
\begin{aligned}
\operatorname{Pr} & \left\{\mathrm{A}_{n} \mid \mathrm{A}_{0} \mathrm{~A}_{1} \ldots \mathrm{A}_{n-1}\right\} \\
& =\operatorname{Pr}\left\{D_{n}(t) \leq \frac{r\left(E_{n}^{\text {init }}-E_{\mathrm{th}}\right)}{\left(2 P_{n}^{\mathrm{e}}+P_{n}^{\mathrm{t}}\right) K L} \mid \mathrm{A}_{0} \mathrm{~A}_{1} \ldots \mathrm{A}_{n-1}\right\} \\
& \approx \operatorname{Pr}\left\{M(t) \leq \frac{r\left(E_{n}^{\text {init }}-E_{\mathrm{th}}\right)}{\left(2 P_{n}^{\mathrm{e}}+P_{n}^{\mathrm{t}}\right) K L R_{0-n}^{\mathrm{L}}} \mid \mathrm{A}_{0} \mathrm{~A}_{1} \ldots \mathrm{A}_{n-1}\right\} \\
& =\sum_{i=0}^{M_{n}^{\prime}} \operatorname{Pr}\left\{M(t)=i \mid \mathrm{A}_{0} \mathrm{~A}_{1} \ldots \mathrm{A}_{n-1}\right\} \\
& =\sum_{k=0}^{M_{n}^{\prime}} \frac{(\Lambda(t))^{k}}{k !} \exp (-\Lambda(t)),
\end{aligned}
$$

where

$$
M_{n}^{\prime}=\left\lfloor\frac{r\left(E_{n}^{\mathrm{init}}-E_{\mathrm{th}}\right)}{\left(2 P_{n}^{\mathrm{e}}+P_{n}^{\mathrm{t}}\right) K L R_{0-n}^{\mathrm{L}}}\right\rfloor .
$$

Using the upper incomplete gamma function defined in [26], (56) can be further modified into

$$
\operatorname{Pr}\left\{\mathrm{A}_{n} \mid \mathrm{A}_{0} \mathrm{~A}_{1} \ldots \mathrm{A}_{n-1}\right\} \approx \Gamma\left(M_{n}^{\prime}+1, \Lambda(t)\right) .
$$

According to the relationship between the Poisson distribution and normal distribution in [30], when the value of $\Lambda(t)$ is sufficiently large, (56) can be expressed approximately as

$$
\begin{aligned}
\operatorname{Pr}\left\{\mathrm{A}_{n} \mid \mathrm{A}_{0} \mathrm{~A}_{1} \ldots \mathrm{A}_{n-1}\right\} & \approx \Phi\left\{\left(\frac{M_{n}^{\prime}+0.5-\Lambda(t)}{\sqrt{\Lambda(t)}}\right)\right\} \\
& =\frac{1}{2}\left[1+\operatorname{erf}\left(\frac{M_{n}^{\prime}+0.5-\Lambda(t)}{\sqrt{2 \Lambda(t)}}\right)\right] .
\end{aligned}
$$

Thus, Proposition 2 is proved.

\section{Competing interests}

The authors declare that they have no competing interests.

\section{Acknowledgements}

This work is supported by National 863 High Technology Development Project (no. 2013AA013601), Key Special Project of National Science and Technology (no. 2013ZX03003006), National Nature Science Foundation of China (nos. $61372106,61102068,61172077$, and 61223001), Research Fund of National Mobile Communications Research Laboratory, Southeast University (no. 2013A04), Program Sponsored for Scientific Innovation Research of College Graduate in Jiangsu Province (no. CXZZ13_0098), Research Fund for the Doctoral Program of Higher Education (no. 20113218120017), and Open Research Fund of National Mobile Communications Research Laboratory, Southeast University (no. 2012D14).

\section{Author details}

${ }^{1}$ National Mobile Communications Research Laboratory, Southeast University, Nanjing 211111, China. ${ }^{2}$ Key Laboratory for Information System Security of Ministry of Education, Tsinghua University, Beijing 100084, China. ${ }^{3}$ Ministry of Education Key Laboratory of Contemporary Design and Integrated Manufacturing Technology, Northwestern Polytechnical University, Xi'an 710072, China. ${ }^{4}$ School of Management and Economics, Beijing Institute of Technology, Beijing 100081, China. ${ }^{5}$ North Information Control Group Co., Ltd., Nanjing 211153, China. ${ }^{6}$ College of Communications Engineering, PLA University of Science and Technology, Nanjing 210007, China.

\section{Received: 12 August 2013 Accepted: 23 October 2013}

Published: 5 December 2013

\section{References}

1. IF Akyildiz, W Su, Y Sankarasubramaniam, E Cayirci, A survey on sensor networks. IEEE Commun. Mag. 40(8), 102-114 (2002)

2. MN Nguyen, C Bao, KL Tew, S Teddy, XL Li, Ensemble based real-time adaptive classification system for intelligent sensing machine diagnostics. IEEE Trans. Reliability 61(2), 303-313 (2012)

3. J Li, $\mathrm{H} \mathrm{Kao}, \mathrm{J} \mathrm{Ke}$, Voronoi-based relay placement scheme for wireless sensor networks. IET Commun. 3(4), 530-538 (2009)

4. J Li, G AlRegib, Network lifetime maximization for estimation in multihop wireless sensor networks. IEEE Trans. Signal Process. 57(7), 2456-2466 (2009)

5. N Wang, XL Shen, Research on WSN nodes location technology in coal mine, in International Forum on Computer Science-Technology and Applications, vol. 3, (IEEE, Chongqing, China, 2009), pp. 232-234

6. J Bredin, E Demaine, M Hajiaghayi, D Rus, Deploying sensor networks with guaranteed fault tolerance. IEEE/ACM Trans. Netw. 18(1), 216-228 (2010)

7. K Sun, P Ning, C Wang, Fault-tolerant cluster-wise clock synchronization for wireless sensor networks. IEEE Trans. Dependable and Sec. Comput. 2(3), 177-189(2005)

8. D Fontanelli, D Petri, An algorithm for WSN clock synchronization: uncertainty and convergence rate trade off, in IEEE International Workshop on Advanced Methods for Uncertainty Estimation in Measurement (IEEE, Bucharest, 2009), pp. 74-79

9. D Ho, S Shimamoto, Highly reliable communication protocol for WSN-UAV system employing TDMA and PFS scheme, in IEEE GLOBECOM Workshops (IEEE, Houston, 2011), pp. 1320-1324

10. X Chen, M Lyu, Reliability analysis for various communication schemes in wireless CORBA. IEEE Trans. Reliability 54(2), 232-242 (2005)

11. JL Cook, JE Ramirez-Marquez, Two-terminal reliability analyses for a mobile ad hoc wireless network. Reliability Eng. Syst. Safety 92(6), 821-829 (2007)

12. JL Cook, JE Ramirez-Marquez, Mobility and reliability modeling for a mobile ad hoc network. IIE Trans. 41(1), 23-31 (2008)

13. JL Cook, JE Ramirez-Marquez, Reliability analysis of cluster-based ad-hoc networks. Reliability Eng. Syst. Safety 93(10), 1512-1522 (2008)

14. S Dominiak, N Bayer, J Habermann, V Rakocevic, B Xu, Reliability analysis of IEEE 802.16 mesh networks, in 2nd IEEE/FIP International Workshop on Broadband Convergence Networks (IEEE, Munich, 2007), pp. 1-12

15. J Liu, X Jiang, Kato Nishiyama H N, Reliability assessment for wireless mesh networks under probabilistic region failure model. IEEE Trans. Vehicular Technol. 60(5), 2253-2264 (2011) 
16. G Egeland, P Engelstad, The availability and reliability of wireless multi-hop networks with stochastic link failures. IEEE J. Select. Areas Commun. 27(7), 1132-1146 (2009)

17. T Kurp, R Gao, S Sah, An adaptive sampling scheme for improved energy utilization in wireless sensor networks, in IEEE Instrumentation and Measurement Technology Conference (IEEE, Austin, 2010), pp. 93-98

18. H AboEIFotoh, E EIMallah, H Hassanein, On the reliability of wireless sensor networks, in IEEE International Conference on Communications, vol. 8, (IEEE, Istanbul, 2006), pp. 3455-3460

19. M Shazly, E Elmallah, H AboEIFotoh, A three-state node reliability model for sensor networks, in IEEE Global Telecommunications Conference (IEEE, Miami, December 2010)

20. BC Cheng, HH Yeh, PH Hsu, Schedulability analysis for hard network lifetime wireless sensor networks with high energy first clustering. IEEE Trans. Reliability 60(3), 675-688 (2011)

21. HC Wong, JMS Nogueira, AAF Loureiro, Fault management in event-driven wireless sensor networks, in the 7th ACM International Symposium on Modeling, Analysis and Simulation of Wireless and Mobile Systems (ACM, Venice, 2004), pp. 149-156

22. RK Shakya, YN Singh, NK Verma, Optimizing channel access for event-driven wireless sensor networks: analysis and enhancements. Arxiv preprint arXiv:1203.5874 (2012)

23. H Luo, H Tao, H Ma, S Das, Data fusion with desired reliability in wireless sensor networks. IEEE Trans. Paral. Distributed Syst. 22(3), 501-513 (2011)

24. H Pham, System Software Reliability (Springer, New York, 2006)

25. TS Rappaport, Wireless Communications: Principles and Practice (Prentice Hall, New Jersey, 1996)

26. F Haight, Handbook of the Poisson Distribution (Wiley, New York, 1967)

27. A Benjamin, A Benjamin, J Quinn, Proofs That Really Count: The Art of Combinatorial Proof (The Mathematical Association of America, Washington, 2003)

28. Binomial distribution. (Wikipedia, 2013), http://en.wikipedia.org/wiki/ Binomial_distribution

29. Beta function. (Wikipedia, 2013), http://en.wikipedia.org/wiki/ Beta_function\#Incomplete_beta_function

30. Poisson distribution. (Wikipedia, 2013), http://en.wikipedia.org/wiki/ Poisson_distribution\#cite_note-Garwood1936--10

doi:10.1186/1687-1499-2013-277

Cite this article as: Wang et al:: Reliability analysis for a data flow in eventdriven wireless sensor networks using a multiple sending transmission approach. EURASIP Journal on Wireless Communications and Networking 2013 2013:277.

\section{Submit your manuscript to a SpringerOpen ${ }^{\circ}$ journal and benefit from:}

- Convenient online submission

- Rigorous peer review

- Immediate publication on acceptance

- Open access: articles freely available online

- High visibility within the field

- Retaining the copyright to your article

Submit your next manuscript at $\boldsymbol{\nabla}$ springeropen.com 